\title{
TITLE:
}

\section{Is the brick-wall model unstable for a rotating background?}

AUTHOR(S):

Mukohyama, S

CITATION:

Mukohyama, S. Is the brick-wall model unstable for a rotating background?. PHYSICAL REVIEW D 2000, 61(12): 124021.

ISSUE DATE:

2000-06-15

URL:

http://hdl.handle.net/2433/50457

RIGHT:

Copyright 2000 American Physical Society 
PHYSICAL REVIEW D, VOLUME 61, 124021

\title{
Is the brick-wall model unstable for a rotating background?
}

\author{
Shinji Mukohyama \\ Yukawa Institute for Theoretical Physics, Kyoto University, Kyoto 606-8502, Japan \\ and Department of Physics and Astronomy, University of Victoria, Victoria, BC, Canada V8W 3P6
}

(Received 5 October 1999; published 26 May 2000)

\begin{abstract}
The stability of the brick-wall model is analyzed in a rotating background. It is shown that, in the Kerr background without an horizon but with an inner boundary, a scalar field has complex-frequency modes and that, however, the imaginary part of the complex frequency can be small enough compared with the Hawking temperature if the inner boundary is sufficiently close to the horizon, say at a proper altitude of Planck scale. Hence the time scale of the instability due to the complex frequencies is much longer than the relaxation time scale of the thermal state with the Hawking temperature. Since ambient fields should settle in the thermal state in the latter time scale, the instability is not so catastrophic. Thus the brick-wall model is well defined even in a rotating background if the inner boundary is sufficiently close to the horizon.
\end{abstract}

PACS number(s): 04.70.Dy

\section{INTRODUCTION}

Understanding the origin of black hole entropy is one of the most interesting problems in black hole physics. The black hole entropy is given by the Bekenstein-Hawking formula $[1,2]$ as

$$
S_{B H}=\frac{1}{4} A_{H},
$$

where $A_{H}$ is area of the horizon.

It seems that a full understanding of black hole entropy requires a theory of quantum gravity, which we do not know yet. However, we believe that the general features of black hole entropy can be understood by semiclassical theory, namely, quantum field theory in a fixed gravitational background. In fact, the brick-wall model proposed by 't Hooft [3] succeeded in deriving the proportionality of black hole entropy to the horizon area by identifying the black hole entropy with the thermal entropy of ambient quantum fields raised to the Hawking temperature. It was recently clarified that in this model back reaction is small enough and that this model is actually a self-consistent model as a semiclassical theory [4]. Moreover, it was shown that this model seeks the maximal value of entanglement entropy in the space of states whose back reaction is small enough [5].

Originally, the brick-wall model is proposed in the spherically symmetric, static background, say, the Schwarzschild background. Hence, it seems interesting to see how this model is extended to a rotating background, say, the Kerr background [6-9]. However, it is known that in a rapidly rotating spacetime without horizon a field has complexfrequency modes $[10,11]$ and that there is the so called ergoregion instability [12]. Thus, it might be expected that the brick-wall model in rotating background might be unstable and unsuitable for the origin of black hole entropy.

In this paper we analyze the stability of the brick-wall model in a rotating background. We show that the time scale of the ergoregion instability is much longer than the relaxation time scale of the thermal state with the Hawking temperature. In the latter time scale ambient fields should settle in the thermal state. Thus, the brick-wall model is well defined even in a rotating background.

In Sec. II we summarize a quantum field theory of a real scalar field in an $n$-dimensional axisymmetric stationary spacetime to show how the appearance of complexfrequency modes alters the structure of the quantum field theory. In Sec. III we consider a scalar field in the 4-dimensional Kerr spacetime without horizon but with an inner boundary to show the existence and a property of the complex frequency. Section IV is devoted to summarizing this paper.

\section{SCALAR FIELD IN ROTATING BACKGROUND}

Let us consider a general $n$-dimensional axisymmetric stationary spacetime $\mathcal{M}$, whose metric is given by

$$
d s^{2}=-N^{2} d t^{2}+\rho^{2}\left(d \varphi-\omega_{B} d t\right)^{2}+q_{a b} d x^{a} d x^{b},
$$

where $a, b=1,2, \ldots,(n-2)$. Here, the lapse function $N$, the Bardeen angular velocity (or minus the $\varphi$-component of the shift vector) $\omega_{B},(\varphi \varphi)$-component $\rho^{2}$ of the metric and the $(n-2)$-dimensional metric $q_{a b}$ are assumed to depend only on the $(n-2)$-dimensional coordinates $\left\{x^{a}\right\}$. On this background spacetime we consider a real scalar field $\phi$ described by the action

$$
I=-\frac{1}{2} \int_{\mathcal{M}} d^{n} x \sqrt{-g}\left(g^{\mu \nu} \partial_{\mu} \phi \partial_{\nu} \phi+\mu^{2} \phi^{2}\right),
$$

where the mass $\mu$ of the field can depend only on the ( $n$ -2)-dimensional coordinates $\left\{x^{a}\right\}$. We impose the boundary condition

$$
N \rho \phi n^{\mu} \partial_{\mu} \phi=0 \quad \text { on } \quad \partial \mathcal{M}
$$

where the boundary $\partial \mathcal{M}$ of the spacetime $\mathcal{M}$ is supposed to be invariant under translations generated by Killing vectors $\partial_{t}$ and $\partial_{\varphi}$, and $n^{\mu}$ is a unit normal to $\partial \mathcal{M}$. Note that a part of $\partial \mathcal{M}$ can be taken at spatial infinity.

In this paper we quantize the system of the scalar field with respect to the time evolution vector $D$ defined by 


$$
D=\left(\frac{\partial}{\partial t}\right)_{\varphi, x^{a}}+\Omega\left(x^{a}\right)\left(\frac{\partial}{\partial \varphi}\right)_{t, x^{a}},
$$

where $\Omega\left(x^{a}\right)$ is an arbitrary function of $\left\{x^{a}\right\}$ such that $D$ is timelike in $\mathcal{M}$. For this choice of the time evolution vector, it is convenient to use a new coordinate system $\left(t, \tilde{\varphi}, x^{a}\right)$ defined by

$$
\tilde{\varphi}=\varphi-\Omega\left(x^{a}\right) t
$$

since for this coordinate system

$$
D=\left(\frac{\partial}{\partial t}\right)_{\tilde{\varphi}, x^{a}} .
$$

Following the usual quantization procedure, it turns out that the canonical momentum $\pi$ conjugate to $\phi$ and, thus, the equal-time commutation relations are independent of the choice of $\Omega\left(x^{a}\right)$ :

$$
\begin{aligned}
\pi & \equiv \frac{\delta L}{\delta(D \phi)}=\frac{\rho \sqrt{q}}{N}\left[D \phi+\left(\omega_{B}-\Omega\right)\left(\frac{\partial \phi}{\partial \tilde{\varphi}}\right)_{t, x^{a}}\right] \\
& =\frac{\rho \sqrt{q}}{N}\left[\left(\frac{\partial \phi}{\partial t}\right)_{\varphi, x^{a}}+\omega_{B}\left(\frac{\partial \phi}{\partial \varphi}\right)_{t, x^{a}}\right],
\end{aligned}
$$

where the Lagrangian $L$ is defined by $I=\int d t L$. Therefore, the quantization procedure we follow is independent of the choice of the time evolution vector $D$. In this sense there is no ambiguity in the quantization.

Off course, there is much freedom in selecting a ground state: we have freedom in the choice of a set of positivefrequency mode functions. In the following, we give one example of the choice of the set of positive-frequency mode functions by using a separation of variables. Other choices give different ground states. However, the Hilbert space of all quantum state is independent of the choice of the set. For example, one ground state can be expressed as excited states above other ground states.

To quantize the system of the scalar field we raise the field $\phi$ to an operator and decompose it by mode functions:

$$
\phi=\sum_{(\omega l m) \in P}\left(\Phi_{\omega l m} a_{\omega l m}+\Phi_{\omega l m}^{*} a_{\omega l m}^{\dagger}\right)
$$

where the set $P$ and the mode functions $\left\{\Phi_{\omega l m}\right\}$ will be defined below by Eq. (2.16) and Eq. (2.17), respectively.

In order to define the mode functions $\left\{\Phi_{\omega l m}\right\}$ in the above expansion, let us seek solutions $\left\{\Psi_{\omega l m}\right\}$ of the field equation by the following separation of variables:

$$
\Psi_{\omega l m}=f_{\omega l m}\left(x^{a}\right) e^{-i \omega t} e^{i m \varphi} .
$$

The function $f_{\omega l m}\left(x^{a}\right)$ is a solution of the equation

$$
\begin{aligned}
& \frac{1}{N \rho \sqrt{q}} \partial_{a}\left(N \rho \sqrt{q} q^{a b} \partial_{b} f_{\omega l m}\right)+\left[\frac{\left(\omega-\omega_{B} m\right)^{2}}{N^{2}}-\frac{m^{2}}{\rho^{2}}-\mu^{2}\right] f_{\omega l m} \\
& \quad=0
\end{aligned}
$$

with the boundary condition

$$
N \rho f_{\omega l m} n^{\mu} \partial_{\mu} f_{\omega^{\prime} l m}=0 \quad \text { on } \quad \partial \mathcal{M}
$$

where $n^{\mu}$ denotes a unit normal to the boundary $\partial \mathcal{M}$. (A part of $\partial \mathcal{M}$ can be taken at spatial infinity.) Here note that, because of the invariance of Eq. (2.10) under $(\omega, m) \leftrightarrow$ $(-\omega,-m)$, we can assume that

$$
f_{\omega l m}^{*}=f_{-\omega^{*}, l,-m} .
$$

We can choose the quantum number $l$ so that

$$
\int d^{n-2} x \frac{\rho \sqrt{q}}{N}\left(\omega-\omega_{B} m\right) f_{\omega l m} f_{\omega^{*} l^{\prime} m}^{*}=0 \quad \text { unless } \quad l=l^{\prime} .
$$

With this choice of the quantum number $l$, the following property holds.

$$
\left(\Psi_{\omega l m}, \Psi_{\omega^{\prime} l^{\prime} m^{\prime}}\right)_{K G}=0
$$

unless

$$
\omega^{*}=\omega^{\prime}, \quad l=l^{\prime} \text { and } m=m^{\prime},
$$

where the Klein-Gordon norm $(\Phi, \Psi)_{K G}$ is given by

$$
(\Phi, \Psi)_{K G}=-i \int d^{n-1} x \frac{\rho \sqrt{q}}{N}\left(\Phi D \Psi^{*}-\Psi^{*} D \Phi\right) .
$$

Now the set $P$, over which the summation is taken in Eq. (2.8), is defined as

$$
\begin{aligned}
P & =P_{R} \cup P_{C}, \\
P_{R} & =\left\{(\omega l m) \mid \omega \text { is real, }\left(\Psi_{\omega l m}, \Psi_{\omega l m}\right)_{K G}>0\right\}, \\
P_{C} & =\{(\omega l m) \mid \Im \omega>0\} .
\end{aligned}
$$

The mode functions $\left\{\Phi_{\omega l m}\right\}$ in the expansion (2.8) are defined by

$$
\begin{aligned}
& \Phi_{\omega l m}=\frac{1}{\sqrt{C_{\omega l m}}} \Psi_{\omega l m} \text { for } \quad(\omega l m) \in P_{R}, \\
& \Phi_{\omega l m}=\frac{1}{\sqrt{2 C_{\omega l m}}}\left(\Psi_{\omega l m}+e^{i \alpha_{\omega l m}} \Psi_{\omega} * l m\right) \text { for } \quad(\omega l m) \in P_{C},
\end{aligned}
$$

where the real constants $C_{\omega l m}(>0)$ and $\alpha_{\omega l m}$ are defined by

$$
\left(\Psi_{\omega l m}, \Psi_{\omega * l m}\right)_{K G}=C_{\omega l m} e^{i \alpha_{\omega l m}}
$$


For the above definition of $P$ and $\left\{\Phi_{\omega l m}\right\}$, the following property can be easily derived:

$$
\begin{aligned}
& \left(\Phi_{\omega l m}, \Phi_{\omega^{\prime} l^{\prime} m^{\prime}}\right)_{K G}=\delta_{\omega \omega^{\prime}} \delta_{l l^{\prime}} \delta_{m m^{\prime}}, \\
& \left(\Phi_{\omega l m}, \Phi_{\omega^{\prime} l^{\prime} m^{\prime}}^{*}\right)_{K G}=0, \\
& \left(\Phi_{\omega l m}^{*}, \Phi_{\omega^{\prime} l^{\prime} m^{\prime}}^{*}\right)_{K G}=-\delta_{\omega \omega^{\prime}} \delta_{l l^{\prime}} \delta_{m m^{\prime}},
\end{aligned}
$$

for $\forall(\omega l m) \in P$ and $\forall\left(\omega^{\prime} l^{\prime} m^{\prime}\right) \in P$. It is these properties that lead us to the above definition of $P$ and $\left\{\Phi_{\omega l m}\right\}$. In Appendix A, it is shown that the local integrability of Eq. (2.24) below requires Eq. (2.20) and that the normalizability of the ground state $|0\rangle$ requires the left hand side of Eq. (2.19) to be positive definite as a matrix with arguments $\lambda$ $=(\omega l m)$ and $\lambda^{\prime}=\left(\omega^{\prime} l^{\prime} m^{\prime}\right)$. [Equation (2.21) is an immediate consequence of Eq. (2.19).]

Since the above orthonormality of the mode functions imply

$$
\left[a_{\lambda}, a_{\lambda^{\prime}}^{\dagger}\right]=\delta_{\lambda \lambda^{\prime}}, \quad\left[a_{\lambda}, a_{\lambda^{\prime}}\right]=0,
$$

the Hilbert space $\mathcal{F}$ of all quantum states can be constructed as a symmetric Fock space spanned by the states $\left|\left\{N_{\lambda}\right\}\right\rangle$ defined by

$$
\left|\left\{N_{\lambda}\right\}\right\rangle=\prod_{\lambda \in P} \frac{\left(a_{\lambda}^{\dagger}\right)^{N_{\lambda}}}{\sqrt{N_{\lambda} !}}|0\rangle,
$$

where the ground state $|0\rangle$ is defined by

$$
a_{\lambda}|0\rangle=0 \quad \text { for } \quad \forall \lambda \in P .
$$

Hereafter, $\lambda$ denotes $(\omega l m)$ and $\bar{\lambda}$ denotes $\left(-\omega^{*}, l,-m\right)$.

The canonical Hamiltonian $H[D]$ with respect to the time evolution vector $D$ is given by

$$
H[D]=\frac{i}{2}(D \phi, \phi)_{K G} .
$$

Hence, if $\Omega$ is a constant, ${ }^{1}$ then $H[D]$ is a conserved quantity and can be expressed as

$$
\begin{aligned}
H[D]= & \frac{1}{2} \sum_{\lambda \in P}\left[(\Re \omega-\Omega m)\left(a_{\lambda} a_{\lambda}^{\dagger}+a_{\lambda}^{\dagger} a_{\lambda}\right)\right. \\
& \left.+i \Im \omega\left(a_{\lambda}^{\dagger} a_{\lambda}^{\dagger}-a_{\lambda} a_{\bar{\lambda}}\right)\right] .
\end{aligned}
$$

Note that any states of the form (2.23) are not eigenstates of this Hamiltonian unless all $\omega$ are real. [Off course, if there is no complex $\omega$ then all states of the form (2.23) are eigenstates of this Hamiltonian.] Moreover, in Appendix B it is shown that there is no ground state suitable for this Hamiltonian unless all $\omega$ are real. To be precise, it is always impossible to eliminate terms including $a_{\lambda}^{\dagger} a_{\lambda}^{\dagger}$ or $a_{\lambda} a_{\lambda}^{-}$in Eq.

\footnotetext{
${ }^{1}$ If $\Omega$ is not a constant then $H[D]$ is not a conserved quantity in general since $\Omega \phi$ does not satisfy the equation of motion.
}

(2.26) by a Bogoliubov transformation. (See Appendix B.) Thus, if there is a complex $\omega$ then there is no stable ground state in $\mathcal{F}$. Hence, existence of complex-frequency modes imply a kind of instability in quantum field theory. This conclusion is consistent with the results of Refs. $[13,12]$ that spectrum of the Hamiltonian becomes continuous and that eigenstates are not normalizable if there is a complexfrequency mode.

Off course, there is a corresponding instability in classical theory: if there is a complex frequency with positive imaginary part then a solution expressed as Eq. (2.9) grows exponentially in time. On the other hand, if imaginary part of frequency is negative then the corresponding solution decays in time but grows exponentially in inverse time.

Therefore, in both classical and quantum senses, appearance of complex-frequency modes implies instability of the system. In the next section, we show that in the brick-wall model a scalar field has complex-frequency modes. Hence, it might be expected that the brick wall model might be unstable and that it might be an unsuitable model to seek entropy for an equilibrium state. However, it turns out that the imaginary part of the complex frequency can be made arbitrarily small by making the inner boundary close enough to the horizon. In fact, in the next section, we show that the imaginary part is small enough compared with the Hawking temperature if the inner boundary is sufficiently close to the horizon, say at a proper altitude of Planck scale.

\section{COMPLEX FREQUENCY MODES AND STABILITY OF THE BRICK-WALL MODEL}

For simplicity, let us consider the (4-dimensional) Kerr spacetime as a background and suppose that the mass $\mu$ is a nonzero constant. The metric is given by

$$
\begin{aligned}
d s^{2}= & -\left(1-\frac{2 M r}{\Sigma}\right) d t^{2}+\frac{\Sigma}{\Delta} d r^{2}+\Sigma d \theta^{2}+R^{2} \sin ^{2} \theta d \varphi^{2} \\
& -\frac{4 M a r}{\Sigma} \sin ^{2} \theta d \varphi d t
\end{aligned}
$$

where

$$
\begin{gathered}
\Sigma=r^{2}+a^{2} \cos ^{2} \theta, \\
\Delta=r^{2}+a^{2}-2 M r, \\
\Sigma R^{2}=\left(r^{2}+a^{2}\right)^{2}-a^{2} \Delta \sin ^{2} \theta .
\end{gathered}
$$

This is of the form (2.1) with 


$$
\begin{aligned}
N^{2} & =\frac{\Delta}{R^{2}}, \\
\omega_{B} & =\frac{2 M a r}{\Sigma R^{2}}, \\
\rho^{2} & =R^{2} \sin ^{2} \theta, \\
q^{a b} d x^{a} d x^{b} & =\frac{\Sigma}{\Delta} d r^{2}+\Sigma d \theta^{2} .
\end{aligned}
$$

We only consider the region $r \geqslant r_{0}$ in this spacetime: we impose the Dirichlet boundary condition on the field $\phi$ at $r$ $=r_{0}$. Hereafter we assume that $r_{0}>M+\sqrt{M^{2}-a^{2}}$ : there is no horizon in the region $r \geqslant r_{0}$.

It is well known that in this background Eq. (2.10) becomes separable. In fact, we can find a solution of Eq. (2.10) of the form

$$
f=\frac{u(r)}{\sqrt{r^{2}+a^{2}}} S(\theta)
$$

where $S$ satisfies

$$
\frac{1}{\sin \theta} \frac{d}{d \theta}\left(\sin \theta \frac{d S}{d \theta}\right)+\left[\lambda-a^{2} \mu^{2}\left(y^{2}-1\right) \sin ^{2} \theta-\frac{m^{2}}{\sin ^{2} \theta}\right] S=0,
$$

and the equation for $u$ can be written as

$$
\frac{d^{2} u}{d x^{2}}+\left(y-V_{+}\right)\left(y-V_{-}\right) u=0
$$

by introducing the nondimensional tortoise coordinate $x$ by

$$
\mu^{-1} \frac{d x}{d r}=\frac{r^{2}+a^{2}}{\Delta}
$$

or

$$
\mu^{-1} x=r+\frac{M}{\sqrt{M^{2}-a^{2}}}\left[r_{+} \ln \left(\frac{r-r_{+}}{r_{+}-r_{-}}\right)-r_{-} \ln \left(\frac{r-r_{-}}{r_{+}-r_{-}}\right)\right] .
$$

Here $r_{ \pm}=M \pm \sqrt{M^{2}-a^{2}}, y \equiv \omega / \mu$ and $V_{ \pm}$are defined by

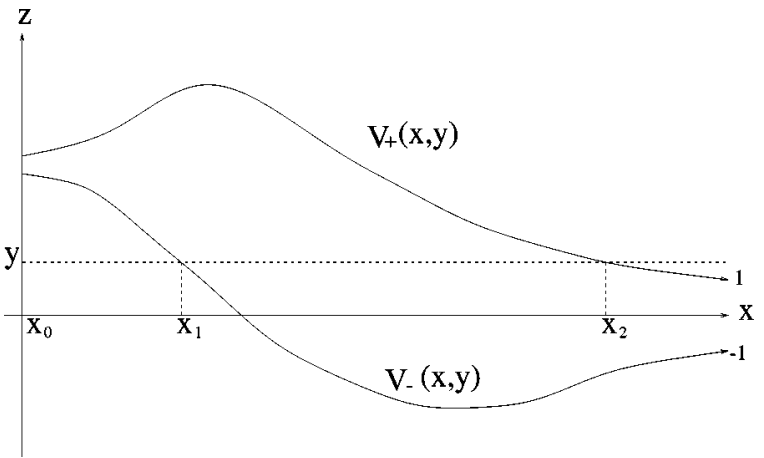

FIG. 1. The typical form of the graphs $z=V_{ \pm}(x, y)$ is written on a fixed $y$ plane. Note that $V_{ \pm}$depend on $y$ as well as $x$ through the eigenvalue $\lambda$ of Eq. (3.5). However, asymptotic behavior of $V_{ \pm}$in the limit $x \rightarrow \pm \infty$ does not depend on $y$.

$$
\begin{aligned}
\mu V_{ \pm}= & \frac{2 m a M r}{\left(r^{2}+a^{2}\right)^{2}} \pm \sqrt{\frac{\Delta \tilde{\mu}^{2}}{r^{2}+a^{2}}}, \\
\tilde{\mu}^{2}= & \mu^{2}+\frac{\lambda}{\left(r^{2}+a^{2}\right)}-\frac{m^{2} a^{2}\left(r^{2}+a^{2}+2 M r\right)}{\left(r^{2}+a^{2}\right)^{3}} \\
& +\frac{2 M r+a^{2}}{\left(r^{2}+a^{2}\right)^{2}}-\frac{6 M a^{2} r}{\left(r^{2}+a^{2}\right)^{3}} .
\end{aligned}
$$

Note that in the horizon limit $r \rightarrow r_{+}($or $x \rightarrow-\infty)$ both of $V_{ \pm}$ approach the same value

$$
V_{ \pm} \rightarrow \frac{m a}{2 \mu M r_{+}}
$$

and that $V_{ \pm}$approaches \pm 1 , respectively, in the limit $r$ $\rightarrow \infty($ or $x \rightarrow \infty)$. (See Fig. 1.)

Now let us seek complex frequency modes by examining a scattering amplitude for real-frequency waves. The method we shall use here is based on the following expected form of the scattering amplitude $S$ near a pole $y=y_{R}+i y_{I}$, providing that $\left|y_{R}\right| \gg\left|y_{I}\right|:[11]$ :

$$
S=e^{2 i \delta_{0}} \times \frac{y-y_{R}+i y_{I}}{y-y_{R}-i y_{I}}
$$

where $\delta_{0}$ is a constant phase. Hence, if we can obtain this form of a scattering amplitude by analyzing real-frequency waves then we find an outgoing normal mode corresponding to $y=y_{R}+i y_{I}$ and an incoming normal mode corresponding to $y=y_{R}-i y_{I}$. After that we should confirm whether these normal modes converge or diverge in the limit of $x \rightarrow \infty$. If these normal modes converge then they give complexfrequency mode functions.

We first consider the case in which $m a>2 \mu M r_{+}$and examine the following five regime separately: (i) $1<y$ $<V_{-}\left(x_{0}\right)$; (ii) $y>V_{+}\left(x_{0}\right)$; (iii) $y<-1$; (iv) $V_{-}\left(x_{0}\right) \leqslant y$ $\leqslant V_{+}\left(x_{0}\right) ;(\mathrm{v})-1 \leqslant y \leqslant 1$.

(i) $1<y<V_{-}\left(x_{0}\right)$ 
In this regime, let the solution of $y=V_{-}$and that of $y$ $=V_{+}$be $x=x_{1}(y)$ and $x=x_{2}(y)$, respectively. (See Fig. 1.) In each region separated by $x_{1}$ and $x_{2}$, the WKB solution is given by

$$
\begin{aligned}
& u=\frac{C_{1}}{|T|^{1 / 4}} \sin \left(\int_{x_{0}}^{x} \sqrt{|T|} d x\right) \text { for } \quad x_{0}<x<x_{1}, \\
& u=\frac{C_{2}}{|T|^{1 / 4}} \exp \left(\int_{x_{1}}^{x} \sqrt{|T|} d x\right)+\frac{C_{3}}{|T|^{1 / 4}} \exp \left(-\int_{x_{1}}^{x} \sqrt{|T|} d x\right)
\end{aligned}
$$$$
\text { for } x_{1}<x<x_{2} \text {, }
$$$$
u=\frac{C_{4}}{|T|^{1 / 4}} \exp \left(i \int_{x_{2}}^{x} \sqrt{|T|} d x\right)+\frac{C_{5}}{|T|^{1 / 4}} \exp \left(-i \int_{x_{2}}^{x} \sqrt{|T|} d x\right)
$$$$
\text { for } x_{2}<x \text {, }
$$

where

$$
T=\left(y-V_{+}\right)\left(y-V_{-}\right) .
$$

Hence, the standard connection formula for WKB solutions gives

$$
S=\frac{C_{4}}{C_{5}}=-i \times \frac{4 e^{2 \eta} \cos \zeta+i \sin \zeta}{4 e^{2 \eta} \cos \zeta-i \sin \zeta},
$$

where

$$
\begin{aligned}
& \zeta=\int_{x_{0}}^{x_{1}} \sqrt{|T|} d x-\frac{\pi}{4}, \\
& \eta=\int_{x_{1}}^{x_{2}} \sqrt{|T|} d x .
\end{aligned}
$$

In the limit $e^{\eta} \rightarrow \infty, S$ approaches to $-i$ unless $\cos \zeta=0$, in which case $S=+i$. Hence, a resonance will occur near a frequency corresponding to $\cos \zeta=0$. We denote the value of $y$ at which

$$
\zeta=\left(n+\frac{1}{2}\right) \pi
$$

by $y_{n}$. Thence, we expand $S$ near $y=y_{n}$ :

$$
S=-i \times \frac{y-y_{n}+i e^{-2 \eta} / 4 \alpha_{n}}{y-y_{n}-i e^{-2 \eta} / 4 \alpha_{n}}+O\left(y-y_{n}\right)^{2},
$$

where

$$
\alpha_{n}=-\left.\frac{d}{d y}\left[\int_{x_{0}}^{x_{1}} \sqrt{|T|} d x\right]\right|_{y=y_{n}}
$$

Because of the behavior (3.10), the asymptotic behavior of $\alpha_{n}$ in the limit of $x_{0} \rightarrow-\infty$ can be obtained: ${ }^{2}$

$$
\alpha_{n} \sim-x_{0} \sim \frac{\mu M r_{+}}{\sqrt{M^{2}-a^{2}}}\left|\ln \left(\frac{r_{0}-r_{+}}{r_{+}-r_{-}}\right)\right|=\frac{\mu}{2 \kappa}\left|\ln \left(\frac{r_{0}-r_{+}}{r_{+}-r_{-}}\right)\right|,
$$

where $\kappa$ is "the surface gravity of the horizon." 3 This implies that $\alpha_{n}>0$. Hence, from Eq. (3.17) and the last of Eq. (3.12), we can conclude that there are two regular solutions, whose asymptotic forms in the limit $x \rightarrow \infty$ are

$$
u \sim \exp \left[\left( \pm i y_{n}-\frac{1}{4 \alpha_{n}} e^{-2 \eta}\right) x\right] .
$$

Here the plus sign corresponds to $S^{-1}=0$ and the minus sign corresponds to $S=0$. Thus, we have obtained a set of complex-frequency modes corresponding to

$$
\omega=\mu\left(y_{n} \pm \frac{i}{4 \alpha_{n}} e^{-2 \eta}\right) .
$$

However, from the asymptotic behavior (3.19) we can conclude that

$$
T_{B H}^{-1} \Im \omega \sim \pm \pi e^{-2 \eta}\left|\ln \left(\frac{r_{0}-r_{+}}{r_{+}-r_{-}}\right)\right|^{-1} \rightarrow 0 \quad\left(r_{0} \rightarrow r_{+}\right),
$$

where $T_{B H}=\kappa / 2 \pi$ is the Hawking temperature of the Kerr background.

(ii) $y>V_{+}\left(x_{0}\right)$

In this regime, analysis depend on how many solutions $y=V_{+}$has. If $y=V_{+}$has no solution or only one degenerate solution then there is no complex frequency modes since whole region, $x_{0} \leqslant x$, is classically allowed region. If $y$ $=V_{+}$has two solutions then we can repeat the above procedure for the regime (i). However, obtained WKB solutions have the asymptotic form (3.20) with negative $\alpha_{n}$ in this case. Thus, there is no regular solutions which correspond to complex-frequency modes.

(iii) $y<-1$

Also in this regime, we can repeat the above procedure. If $y=V_{-}$has no solution or only one degenerate solution then there is no complex frequency mode. For the case in which $y=V_{-}$has two solutions, obtained WKB solution $u$ has the asymptotic form

$$
u \sim \exp \left[\left( \pm i y_{n}+\frac{1}{4 \alpha_{n}} e^{-2 \eta}\right) x\right]
$$

\footnotetext{
${ }^{2}$ Although $\lambda$ in $V_{ \pm}$depends on $y$ through the eigenequation (3.5), this asymptotic behavior of $\alpha_{n}$ is correct since the right hand side of Eq. (3.10) is independent of $\lambda$.

${ }^{3}$ Strictly speaking, in our background there is no horizon by assumption. However, redshifted local acceleration is bounded from above by the surface gravity of the horizon in the extended spacetime which has a horizon.
} 
with positive $\alpha_{n}$. Thus, there is no regular solution which corresponds to complex-frequency modes.

(iv) $V_{-}\left(x_{0}\right) \leqslant y \leqslant V_{+}\left(x_{0}\right)$

In this regime, let the solution of $y=V_{+}$be $x=x_{2}(y)$. In each region separated by $x_{2}$, the WKB solution is given by

$$
\begin{aligned}
& u=\frac{C_{1}}{|T|^{1 / 4}} \sinh \left(\int_{x_{0}}^{x} \sqrt{|T|} d x\right) \text { for } x_{0}<x<x_{2}, \\
& u=\frac{C_{4}}{|T|^{1 / 4}} \exp \left(i \int_{x_{2}}^{x} \sqrt{|T|} d x\right)+\frac{C_{5}}{|T|^{1 / 4}} \exp \left(-i \int_{x_{2}}^{x} \sqrt{|T|} d x\right)
\end{aligned}
$$$$
\text { for } x_{2}<x \text {, }
$$

hence the standard connection formula for WKB solutions gives

$$
S=\frac{C_{4}}{C_{5}}=-i \times \frac{2 e^{2 \eta}-i}{2 e^{2 \eta}+i},
$$

where

$$
\eta=\int_{x_{0}}^{x_{2}} \sqrt{|T|} d x .
$$

From this expression of $S$, it is evident that there is no complex-frequency mode near the real axis.

(v) $-1 \leqslant y \leqslant 1$

In this regime, the region with large $x$ is classically forbidden region. Thus, there is no complex-frequency mode.

Next, let us consider the case in which $m a<-2 \mu M r_{+}$. The above analysis can be applied to this case by simply replacing $y$ with $-y, V_{+}$with $-V_{-}$, and $V_{-}$with $-V_{+}$. Complex frequency modes arise only in the regime $V_{+}\left(x_{0}\right)$ $<y<-1$ and

$T_{B H}^{-1} \Im \omega \sim \pm \pi e^{-2 \eta}\left|\ln \left(\frac{r_{0}-r_{+}}{r_{+}-r_{-}}\right)\right|^{-1} \rightarrow 0 \quad\left(r_{0} \rightarrow r_{+}\right)$.

Finally, let us consider the case in which $-2 \mu M r_{+}$ $\leqslant m a \leqslant 2 \mu M r_{+}$. From the above analysis for other cases, it is evident that there arise no complex-frequency mode functions since neither the regime $1<y<V_{-}\left(x_{0}\right)$ nor the regime $V_{+}\left(x_{0}\right)<y<-1$ exist in this case.

In summary, in this section we have shown that in the Kerr background without horizon but with an inner boundary a scalar field has complex-frequency modes and that the imaginary part of the complex frequency is small enough compared with the Hawking temperature if the inner boundary is sufficiently close to the horizon, say at a proper altitude of Planck scale.

\section{SUMMARY AND DISCUSSION}

We had analyzed the stability of the brick-wall model in a rotating background. We had shown that in the Kerr background without horizon but with an inner boundary a scalar field has complex-frequency modes and that, however, the imaginary part of the complex frequency can be small enough compared with the Hawking temperature if the inner boundary is sufficiently close to the horizon, say at a proper altitude of Planck scale. Hence, the time scale of the ergoregion instability is much longer than the relaxation time scale of the thermal state with the Hawking temperature. In the latter time scale ambient fields should settle in the thermal state. In this sense ergoregion instability is not so catastrophic. Thus, the brick-wall model is well defined if the inner boundary is sufficiently close to the horizon.

Now, let us discuss physical interpretation of the existence of complex-frequency modes. First, for a rotating black hole background, there is no complex frequency mode [14]. However, it is well known that superradiant modes of fields are amplified by scattering. On the other hand, for the brickwall (or a rapidly rotating star) background, the amplification of superradiant modes can be suppressed by a boundary condition say, the Dirichlet boundary condition at the inner boundary [15]. Instead of the amplification of superradiant modes, as shown in this paper, for this background there appear complex frequency modes. These complex frequency modes are outgoing and incoming normal modes of the field and may be understood intuitively as "quasibound states" in the ergoregion. [See Eq. (3.16).] This interpretation is consistent with the fact that for a black hole background there is no complex frequency mode since there is no "quasibound state" in the ergoregion: any excitations with negative energy with respect to observers at infinity will fall into the hole. Based on this observation, thus, it is expected that in the brick-wall background the imaginary part of the complex frequency should become arbitrarily small in the limit that the inner boundary becomes close enough to the horizon since in this limit there appears a large room for the excitations with negative energy with respect to observers at infinity to escape to. This consideration is, of course, consistent with our result in this paper: we have shown that the imaginary part of the complex frequency can be small enough compared with the time scale determined by the Hawking temperature if the inner boundary is sufficiently close to the horizon, say at a proper altitude of Planck scale.

Next, let us discuss a relation to the so-called SchiffSnyder-Weinberg effect [16]. In Ref. [17] the relation between the Klein paradox [18] and superradiance in a rotating black hole background was discussed in detail by using a rectilinear model of the Kerr spacetime. Since the situation in the Klein paradox can be understood as a limit (the socalled Klein limit) of the Schiff-Snyder-Weinberg effect [17], the situation in a rotating black hole background should be understood as a limit of our situation, i.e., the brick-wall model. This is actually the case: in the $x_{0} \rightarrow-\infty$ limit the complex frequencies in the brick-wall background disappear as in the Kerr black hole or in the rectilinear spacetime considered in Ref. [17].

Finally, we would like to mention a possibility to stabilize the quantum field theory in a rotating background by introducing a nonlinear interaction. In Refs. $[19,20]$ it was suggested that a nonlinear interaction will prevent the vacuum from being unstable even if there are complex-frequency modes. It will be interesting to investigate such a possibility 
in the case of quantum field theory in a rotating background. Physics in a rapidly rotating background spacetime will be as interesting as physics of strong fields [21].

\section{ACKNOWLEDGMENTS}

The author would like to thank Professor W. Israel for his warmest hospitality in University of Victoria and many truly stimulating discussions. He also would like to thank Professor H. Kodama for his continuing encouragement. This work was supported partially by the Grant-in-Aid for Scientific Research Fund (No. 9809228).

\section{APPENDIX A: KLEIN-GORDON NORM AND INTEGRABILITY}

In Sec. II we have expanded the field operator $\phi$ as Eq. (2.8) by mode functions $\left\{\Phi_{\omega l m}\right\}$ satisfying Eqs. (2.19)(2.21). In other words, we have required that coefficients of annihilation operators should have positive Klein-Gordon norm instead of requiring positivity of the frequency $\omega$ with respect to the Killing time $t$.

In this appendix we show that the positivity of the KleinGordon norm is required by integrability of equations for the ground state, say, Eq. (2.24).

Let us consider a scalar field $\phi$ described by the action (2.2) in a general $n$-dimensional globally-hyperbolic spacetime:

$$
d s^{2}=-N^{2} d t^{2}+\gamma_{j k}\left(d x^{j}+\beta^{j} d t\right)\left(d x^{k}+\beta^{k} d t\right),
$$

where the lapse function $N$, the shift vector $\beta^{j}$, the $(n-1)$-dimensional metric $\gamma_{j k}$ and the mass $\mu$ can depend on both $t$ and the $(n-1)$-dimensional coordinates $\left\{x^{j}\right\}$.

To make our arguments definite, let us discretize the system of the scalar field. Since the Lagrangian $L$ defined by $I$ $=\int d t L$ can be written as

$$
\begin{aligned}
L= & \frac{1}{2} \int d^{n-1} x\left[\frac{\sqrt{\gamma}}{N}\left(\partial_{t} \phi-\beta^{j} \partial_{j} \phi\right)^{2}\right. \\
& \left.-N \sqrt{\gamma}\left(\gamma^{j k} \partial_{j} \phi \partial_{k} \phi+\mu^{2} \phi^{2}\right)\right],
\end{aligned}
$$

we can discretize it to obtain ${ }^{4}$

$$
L=\frac{1}{2} G_{A B}\left(\dot{\phi}^{A}-f_{C}^{A} \phi^{C}\right)\left(\dot{\phi}^{B}-f_{D}^{B} \phi^{D}\right)-\frac{1}{2} V_{A B} \phi^{A} \phi^{B}
$$

provided that we suppose the following correspondence:

\footnotetext{
${ }^{4}$ Although explicit forms of the matrices $G, f$ and $V$ depend on the way of discretization, all we need to take the continuous limit in the following arguments is the correspondence (A4) only. Thus, the result in this appendix is independent of an explicit way of discretization.
}

$$
\begin{array}{r}
\int d^{n-1} x \frac{\sqrt{\gamma}}{N}\left(\partial_{t} \phi\right)^{2} \Leftrightarrow G_{A B} \dot{\phi}^{A} \dot{\phi}^{B}, \\
\beta^{j} \partial_{j} \phi \Leftrightarrow f_{C}^{A} \phi^{C}, \\
\int d^{n-1} x N \sqrt{\gamma}\left(\gamma^{j k} \partial_{j} \phi \partial_{k} \phi+\mu^{2} \phi^{2}\right) \Leftrightarrow V_{A B} \phi^{A} \phi^{B} .
\end{array}
$$

These relations will be used when we take a continuous limit. The corresponding equation of motion and the commutation relations are

$$
\begin{aligned}
& \left(G_{A B} \dot{\phi}^{B}-G_{A B} f_{C}^{B} \phi^{C}\right)+G_{B C} f_{A}^{C} \dot{\phi}^{B}+\left(V_{A B}-f_{A}^{C} G_{C D} f_{B}^{D}\right) \phi^{B} \\
& \quad=0
\end{aligned}
$$

and

$$
\left[\phi^{A}, \pi_{B}\right]=i \delta_{B}^{A}, \quad\left[\phi^{A}, \phi_{B}\right]=\left[\pi^{A}, \pi_{B}\right]=0,
$$

where the momentum $\pi_{A}$ conjugate to $\phi^{A}$ is defined by

$$
\pi_{A} \equiv \frac{\partial L}{\partial \dot{\phi}^{A}}=G_{A B}\left(\dot{\phi}^{B}-f_{C}^{B} \phi^{C}\right) .
$$

Now let us expand the field operator by "mode functions" $\left\{\Phi_{n}^{A}, \Phi_{n}^{A *}\right\}(n=1,2, \ldots)$, i.e. solutions of the equation of motion:

$$
\phi^{A}=\sum_{n}\left(a_{n} \Phi_{n}^{A}+a_{n}^{\dagger} \Phi_{n}^{A *}\right)
$$

where we assume that $\left\{\Phi_{n}^{A}, \Phi_{n}^{A *}\right\}$ forms a complete set of linearly independent solutions of the equation of motion.

We would like to define the corresponding ground state by

$$
a_{n}|0\rangle=0, \quad \text { for } \forall n \text {. }
$$

This is the discretized version of Eq. (2.24). However, this equation is not integrable in general. Hence, in this appendix we would like to seek the necessary and sufficient condition for the integrability of this equation.

First, it is easily shown that a certain linear combination of $\phi^{A}$ and $\pi_{A}$ is written as a linear combination of $a_{n}$ as follows:

$$
\pi_{A}-i \Omega_{A B} \phi^{B}=-i \sum_{n} a_{n} \Phi_{n}^{B}\left(\Omega+\Omega^{*}\right)_{A B},
$$

where the matrix $\Omega_{A B}$ is defined by

$$
\Omega_{A B}=-i G_{A C}\left[\left(\Phi^{-1}\right)_{B}^{n *} \dot{\Phi}_{n}^{C *}-f_{B}^{C}\right]
$$

Thus, in order for Eq. (A9) to be integrable, it is necessary that

$$
\begin{aligned}
& {\left[\left(\pi_{A}-i \Omega_{A C} \phi^{C}\right),\left(\pi_{B}-i \Omega_{B D} \phi^{D}\right)\right]|0\rangle=\left(\Omega_{A B}-\Omega_{B A}\right)|0\rangle} \\
& \quad=0
\end{aligned}
$$


which is equivalent to

$$
\begin{aligned}
\Phi_{n}^{A *}\left(\Omega_{A B}-\Omega_{B A}\right) \Phi_{m}^{B *}= & -i G_{A B}\left[\Phi_{n}^{A *}\left(\Phi_{m}^{B *}-f_{C}^{B} \Phi_{m}^{C *}\right)\right. \\
& \left.-\left(\Phi_{n}^{A *}-f_{C}^{A} \Phi_{n}^{C *}\right) \Phi_{m}^{B *}\right]=0
\end{aligned}
$$

Next, by using the relation (A10), the solution of Eq. (A9) is obtained at least locally as follows, provided that the local integrability condition (A13) is satisfied:

$$
\left\langle\left\{\phi^{A}\right\} \mid 0\right\rangle=\mathcal{N} \exp \left[-\frac{1}{2} \Omega_{A B} \phi^{A} \phi^{B}\right] .
$$

In order for this wave function to be well-defined, i.e., normalizable, it is necessary and sufficient that the Hermite matrix $\left(\Omega+\Omega^{\dagger}\right)_{A B}$ be positive definite. This condition is restated that the matrix $X_{n m}$ defined as follows should be positive definite:

$$
\begin{aligned}
X_{n m} & \equiv \Phi_{n}^{A}\left(\Omega+\Omega^{\dagger}\right)_{A B} \Phi_{m}^{B *} \\
& =-i G_{A B}\left[\Phi_{n}^{A}\left(\Phi_{m}^{B *}-f_{C}^{B} \Phi_{m}^{C *}\right)-\left(\Phi_{n}^{A}-f_{C}^{A} \Phi_{n}^{C}\right) \Phi_{m}^{B *}\right] .
\end{aligned}
$$

In summary, in order for Eq. (A9) to be integrable, it is necessary and sufficient that the condition (A13) is satisfied and that the matrix $X_{n m}$ defined by Eq. (A15) is positive definite. These two conditions can be restated as follows:

$$
\begin{gathered}
\left(\Phi_{n}^{*}, \Phi_{m}\right)=0, \text { for } \forall n, m, \\
\left(\Phi_{n}, \Phi_{m}\right) \quad \text { is positive definite, }
\end{gathered}
$$

where the norm $(\Phi, \Psi)$ is defined by

$$
(\Phi, \Psi) \equiv-i G_{A B}\left[\Phi^{A}\left(\Psi^{B *}-f_{C}^{B} \Psi^{C *}\right)-\left(\Phi^{A}-f_{C}^{A} \Phi^{C}\right) \Psi^{B *}\right] .
$$

It is easy to show by using the equation of motion (A5) that this norm is constant in time if both $\Phi$ and $\Psi$ satisfy the equation of motion.

Provided that the condition (A17) is satisfied, it is possible to take linear transformation of $\left\{\Phi_{n}\right\}$ so that

$$
\left(\Phi_{n}, \Phi_{m}\right)=\delta_{n m},
$$

preserving the condition (A16). In this normalization, it can be easily shown that

$$
\left[a_{n}, a_{m}^{\dagger}\right]=\delta_{n m}, \quad\left[a_{n}, a_{m}\right]=\left[a_{n}^{\dagger}, a_{m}^{\dagger}\right]=0 .
$$

Thus, we can construct the Hilbert space of all quantum states spanned by

$$
\left|\left\{N_{n}\right\}\right\rangle=\left(\prod_{n} \frac{\left(a_{n}^{\dagger}\right)^{N_{n}}}{N_{n} !}\right)|0\rangle .
$$

Now let us take a continuous limit. From the correspondence (A4), it is evident that the norm (A18) is a discretized version of the Klein-Gordon norm

$$
\begin{gathered}
(\Phi, \Psi)_{K G}=-i \int d^{n-1} x \sqrt{\gamma} u^{\mu}\left(\Phi \partial_{\mu} \Psi^{*}-\Psi^{*} \partial_{\mu} \Phi\right), \\
u^{\mu} \partial_{\mu}=\frac{1}{N}\left(\partial_{t}-\beta^{j} \partial_{j}\right),
\end{gathered}
$$

which reduces to Eq. (2.15) for a spacetime metric of the form (2.1).

It is also evident that the integrability conditions (A16) and (A19) in the continuous limit for the expansion (2.8) is that Eqs. (2.20) and (2.19) for $\forall(\omega l m) \in P$ and $\forall\left(\omega^{\prime} l^{\prime} m^{\prime}\right) \in P$.

\section{APPENDIX B: HAMILTONIAN FOR A COMPLEX FREQUENCY MODE}

In this appendix, we show that it is always impossible to eliminate terms including $a_{\lambda}^{\dagger} a_{\lambda}^{\dagger}$ or $a_{\lambda} a_{\lambda}^{-}$in Eq. (2.26) by a Bogoliubov transformation.

In general, a Bogoliubov transformation can be written in terms of two matrices $\alpha$ and $\beta$ satisfying

$$
\begin{aligned}
& \alpha \alpha^{\dagger}-\beta \beta^{\dagger}=\mathbf{1}, \\
& \alpha \beta^{T}-\beta \alpha^{T}=0
\end{aligned}
$$

as

$$
\Phi_{n} \rightarrow \sum_{m}\left(\alpha_{n m} \Phi_{m}+\beta_{n m} \Phi_{m}^{*}\right)
$$

where $\left\{\Phi_{n}\right\}(n=1,2, \ldots)$ is a set of positive frequency mode functions. Let us consider a Hamiltonian of the form

$$
H=\frac{1}{2} E^{n m}\left(a_{n} a_{m}^{\dagger}+a_{n}^{\dagger} a_{m}\right)+\frac{1}{2} \Lambda^{n m} a_{n} a_{m}+\frac{1}{2} \Lambda^{n m *} a_{n}^{\dagger} a_{m}^{\dagger},
$$

where $E$ is a Hermite matrix and $\Lambda$ is a symmetric matrix. Under the Bogoliubov transformation, the coefficientmatrices $E$ and $\Lambda$ are transformed as

$$
\begin{aligned}
& E \rightarrow\left(\alpha E \alpha^{\dagger}+\beta E^{*} \beta^{\dagger}\right)+\left(\alpha \Lambda \beta^{\dagger}-\beta \Lambda^{*} \alpha^{\dagger}\right), \\
& \Lambda \rightarrow\left(\alpha \Lambda \alpha^{T}-\beta \Lambda^{*} \beta^{T}\right)+\left(\alpha E \beta^{T}+\beta E^{*} \alpha^{T}\right) .
\end{aligned}
$$

Returning to the problem, the contribution of a pair of complex-frequency modes $\lambda$ and $\bar{\lambda}$ to the Hamiltonian (2.26) can be written as

$$
\begin{aligned}
h= & \frac{1}{2}(\Re \omega-\Omega m)\left[\left(a_{\lambda} a_{\lambda}^{\dagger}+a_{\lambda}^{\dagger} a_{\lambda}\right)-\left(a_{\lambda} a_{\lambda}^{\dagger}+a_{\lambda}^{\dagger} a_{\lambda}^{-}\right)\right] \\
& +i \mathfrak{I} \omega\left(a_{\lambda}^{\dagger} a_{\lambda}^{\dagger}-a_{\lambda} a_{\bar{\lambda}}^{-}\right) .
\end{aligned}
$$

This is of the form (B3) with 


$$
E=(\Re \omega-\Omega m)\left(\begin{array}{cc}
1 & 0 \\
0 & -1
\end{array}\right), \quad \Lambda=-i \Im \omega\left(\begin{array}{ll}
0 & 1 \\
1 & 0
\end{array}\right)
$$

Hence, what we shall show now is that there is no choice of $2 \times 2$ matrices $\alpha$ and $\beta$ satisfying Eq. (B1) and

$$
\left(\alpha \Lambda \alpha^{T}-\beta \Lambda^{*} \beta^{T}\right)+\left(\alpha E \beta^{T}+\beta E^{*} \alpha^{T}\right)=0 .
$$

This statement is easy to show. First, since the first of Eq. (B1) implies that $\alpha$ has the inverse, the second of Eq. (B1) and Eq. (B7) are written as $\gamma=\gamma^{T}$ and

$$
\begin{aligned}
i \mathfrak{I} \omega & \left\{\left(\begin{array}{ll}
0 & 1 \\
1 & 0
\end{array}\right)+\gamma\left(\begin{array}{cc}
0 & 1 \\
1 & 0
\end{array}\right) \gamma^{T}\right\} \\
& =(\Re \omega-\Omega m)\left\{\left(\begin{array}{cc}
1 & 0 \\
0 & -1
\end{array}\right) \gamma^{T}+\gamma\left(\begin{array}{cc}
1 & 0 \\
0 & -1
\end{array}\right)\right\},
\end{aligned}
$$

where $\gamma=\alpha^{-1} \beta$. These equation are easy to solve with respect to $\gamma$. The result gives $|\operatorname{det} \gamma|=1$. However, from the first of Eq. (B1) it is derived that $|\operatorname{det} \gamma|<1$, which contradicts with the above result. Therefore, there is no choice of $2 \times 2$ matrices $\alpha$ and $\beta$ satisfying Eqs. (B1) and (B7).
[1] J.D. Bekenstein, Phys. Rev. D 7, 949 (1973).

[2] S.W. Hawking, Commun. Math. Phys. 43, 199 (1975).

[3] G. 't Hooft, Nucl. Phys. B256, 727 (1985).

[4] S. Mukohyama and W. Israel, Phys. Rev. D 58, 104005 (1998).

[5] S. Mukohyama, Phys. Rev. D 61, 064015 (2000); "Entanglement/Brick-wall entropies correspondence," in General Relativity and Relativistic Astrophysics, Proceedings of the Eighth Canadian Conference, Montreal, Quebec, 1999, edited by C.P. Burgess and R.C. Myers (AIP, New York, 2000).

[6] M.H. Lee and J.K. Kim, Phys. Rev. D 54, 3904 (1996).

[7] J. Ho, W.T. Kim, Y.J. Park, and H. Shin, Class. Quantum Grav. 14, 2617 (1997).

[8] J. Ho and G. Kang, Phys. Lett. B 445, 27 (1998).

[9] V.P. Frolov and D.V. Fursaev, Phys. Rev. D 61, 024007 (2000).

[10] H. Sato and K. Maeda, Prog. Theor. Phys. 59, 1173 (1978).

[11] N. Comins and B.F. Schutz, Proc. R. Soc. London A364, 211
(1978).

[12] G. Kang, Phys. Rev. D 55, 7563 (1997).

[13] B. Schroer and J.A. Swieca, Phys. Rev. D 2, 2938 (1970).

[14] B.F. Whiting, J. Math. Phys. 301, 1301 (1989).

[15] A.L. Matacz, P.C.W. Davies, and A.C. Ottewill, Phys. Rev. D 47, 1557 (1993).

[16] L.I. Schiff, H. Snyder, and J. Weinberg, Phys. Rev. 57, 315 (1940).

[17] S. A. Fulling, Aspects of Quantum Field Theory in Curved Space-Time (Cambridge University Press, Cambridge, England, 1989), Appendix.

[18] O. Klein, Z. Phys. 53, 157 (1929).

[19] A.B. Migdal, Zh. Eksp. Teor. Fiz. 61, 2209 (1971) [Sov. Phys. JETP 34, 1184 (1972)].

[20] A. Klein and J. Rafelski, Phys. Rev. D 11, 300 (1975); 12, 1194 (1975).

[21] Quantum Electrodynamics of Strong Fields, edited by W. Greiner (Plenum Press, New York, 1983). 